\title{
A NEW ALGORITHM FOR OPTIMIZATION OF THE FUZZY RELATION EQUATION WITH MAX- ALGEBRAIC SUM COMPOSITION
}

\author{
V. Zharfi ${ }^{1}$ and A. Mirzazadeh ${ }^{2^{*}}$ \\ 1,2Department of Industrial Engineering \\ University of Tarbiat Moallem (Kharazmi), Tehran, Iran \\ v.zharfi@tmu.ac.ir, a.mirzazadeh@aut.ac.ir
}

\begin{abstract}
This paper considers an optimization problem with a linear objective function under the constraints expressed by a system of fuzzy relation equations using max-as (Algebraic Sum) composition. First, some properties of minimal solutions of the system with fuzzy relation equations and max-as composition are shown. Then, a new algorithm for solving the optimization problem is derived. The numerical examples have been provided to illustrate the theoretical results.
\end{abstract}

\section{OPSOMMING}

Hierdie artikel bestudeer ' $\mathrm{n}$ optimiseringsprobleem met ' $\mathrm{n}$ lineêre doelwitfunksie en wasige randvoorwaardes met ' $n$ algebraïese somsamestelling. Aanvanklik word sommige eienskappe van die minimale oplossings van die wasige vergelykings en die algebraïese samestelling getoon. Daarna word ' $n$ nuwe algoritme vir die oplossing van die optimiseringsprobleem afgelei. Numeriese voorbeelde word voorsien om die teoretiese resultate te ondersteun.

\footnotetext{
${ }^{1}$ The author was enrolled for an MEng (Industrial) degree in the Department of Industrial Engineering, University of Tarbiat Moallem.

${ }^{2}$ The author was enrolled for a DPhil (Engineering Management) degree in the Department of Industrial Engineering, University Tarbiat Moallem.

*Corresponding author.
} 


\section{INTRODUCTION}

The following linear programming model with fuzzy relation constraints has been considered:

Minimize $z(x)=\sum_{i=1}^{m} c_{i} \cdot x_{i}$

Subject to: $X \mathrm{oA}=\mathrm{b} ; \quad 0 \leq \mathrm{x}_{\mathrm{i}} \leq 1$

Where, $\mathrm{c}=\left(\mathrm{c}_{1}, \mathrm{c}_{2}, \ldots, \mathrm{c}_{\mathrm{m}}\right)$ is a m-dimensional vector; $c_{i}$ represents the cost associated with variable $x_{i}$ for $i=1,2, \ldots, m$, and $b=\left(b_{1}, b_{2}, \ldots, b_{n}\right)$ is an n-dimensional vector with $0 \leq b_{j} \leq 1$. The operation " $\mathrm{O}$ " in Eq. (2) is an algebraic composition. The resolution of the fuzzy relation in Eq. (2) is an interesting and ongoing research topic [1], [2], [3], [4], [5]. Many different equations are based on a specific composition. Relations that have been used in these equations include max-min, max-average, max-product, and other fuzzy compositions. Each of these compositions may not be useful in certain special circumstances. For instance, even if we want the fuzzy set with the largest membership to have a major impact on the equation and to use the minimum operator, this would be impossible. Each of these fuzzy operators has a special property as follows:

$\min (a, b)$ is equivalent to the intersection logical operator between them; so " $a$ " and " $b$ " ( $0 \leq a, b \leq 1)$ are fuzzy numbers. The min operator is used for conservative estimates so that a value can compensate for the losses of another one. Zimmermann [5] employed the average operation to represent the "and" fuzzy operator. $a() b=.a . b$ indicates a fuzzy product. This operator has a decreasing effect, and is used in cases where two operatives are going to debilitate each other. $a(+) b=a+b-a . b$ indicates a fuzzy algebraic sum. This operator has an increasing effect, and is used when two operatives are going to reinforce each other. In this paper, the following linear programming model with fuzzy relation constraints has been considered:

Minimize $z(x)=c_{1} \cdot x_{1}+c_{2} \cdot x_{2}+\ldots+c_{m} \cdot x_{m}$

Subject to: $\max _{i}\left(a_{i j}(+) x_{i}\right)=b_{j}, \forall j$

where $0 \leq a_{i j} \leq 1,0 \leq b_{j} \leq 1 \quad(i=1,2, \ldots, m, j=1,2, \ldots, n)$; the operations '.'and '+' are multiplication and addition in Eq. (3) respectively, and $a(+) b=a+b-a \cdot b$ is a fuzzy algebraic sum in the system (4).

The non-empty feasible solution set of fuzzy relation (Eq. (2)) is generally a non-convex set, which can be completely determined by one maximum solution and a finite number of minimum solutions. The fuzzy relation equations are used in many problems such as medical diagnosis [2], system analysis [6], decision-making [3], Fuzzy control [7], Fuzzy modelling [8], and in psychology, economics, sociology, and especially Fuzzy arithmetic [7], [9].

In the first effort in this direction, the fuzzy relation equations were studied by Sanchez [10], considering max-min composition. Later Pedrycz [6] studied max-product fuzzy relation equations. Di Nola et al. [11] showed that the solution set of Eq. (2) can be determined completely by a unique maximum solution and a finite number of minimal solutions [12]. Wu \& Guu [13] proposed the necessary condition for optimal solution of the problems with positive cost coefficients in the objective function. Based on this necessary 
condition, three rules have been applied in simplifying the finding of optimal solutions. However, as mentioned in the first example of this article, in some cases these rules are not effective. Finally, after reducing the size of the problem, the rest of the problem can be solved by a branch and bound method. Although the authors of this paper update the upper bound of the branch and bound method - when a better bound is generated and causes the number of the nodes in this method to be reduced - it still requires a relatively time-consuming process to solve the branch and bound problem. Wu [14] considered the fuzzy relation equation with max-average composition. He proposed six rules that are very effective in reducing the problem size. However, using these rules does not develop an algorithmic framework that can begin from a starting point and add value to all the variables. These rules only reduce the size of the problem. After these rules are implemented, some uncertain variables may have remained; these variables may be resolved using the branch and bound method. Khorram, Ghodousian \& Molai [15] investigated a minimization problem with a linear objective function subjected to fuzzy relation equations with max-as composition. They characterised the feasible solution set and then introduced two efficient procedures for solving the problem. They considered the constraint part of the system (4) which consists of fuzzy relation equations with max-as composition. These scientists decomposed the problem into two sub-problems: the nonnegative, and the non-positive cost coefficients in the objective function. According to their procedure, the sub-problem formed by non-positive coefficients was solved by maximum solution. Then, the variables with non-positive coefficients were removed from the objective function, and two algorithms were proposed to solve the sub-problem. Such problems were formed by the remaining variables, the non-negative coefficients in the objective function under the original constraint. Finally, they combined the two solutions derived from those two sub-problems to yield an optimal solution for (1)-(2) problem. Following the previous studies, the algorithm presented in this paper is a needless heuristic from the traditional methods; and by using the augmented matrix and assigning values to all variables in each step of the algorithm, this will lead to an efficient and complete answer at the end of the stages. High-quality solutions from this algorithm are evident from the examples given at the end of the paper, and can be compared with similar algorithms that deal with this subject [15]. In this research, at first the necessary condition is provided for an optimal solution of (3)-(4) problem. Then an augmented matrix is used to capture the contribution of each variable in the objective function yielded by employing the necessary condition. Finally, a new algorithm is presented for solving (3)-(4) model. As the mentioned matrix and algorithm help to find optimal solutions, the (3)-(4) problem is not decomposed into two sub-problems, and no rules are needed to reduce the problem size.

\section{PRELIMINARY PROPERTIES FOR MAX-AS COMPOSITION}

Let $X=\left\{x \in R^{m}: 0 \leq x_{i} \leq 1, i \in I\right\}, I=\{1,2, . ., m\}$ and $J=\{1,2, . ., n\}$, as the feasible solution of the system (4) is denoted by $X[A, b]=\left\{x \in R^{m}: X \mathrm{o} A=b\right\}$ to characterise $X[A, b] ; \quad \hat{x} \in X[A, b]$ is said to be the maximum solution if $z(x) \leq z(\widehat{x}), \forall x \in X[A, b]$; similarly, $\breve{x} \in X[A, b]$ is the minimum solution; if for $x$ in $X[A, b]$ we have $z(x) \leq z(\widehat{x})$, then $z(x)=z(\widehat{x})$. Here, the following lemmas and theorems are presented, which are required in the subsequent sections.

\subsection{Lemma 1}

If in the $\mathrm{j}$-th equation $a_{i j}>b_{j}$ holds for $i \in I$ in the system (4), then the solution set $X(A, b)$ is empty. 


\subsubsection{Proof}

Suppose that $X[A, b]$ is not empty; therefore we must have: $\max _{i \in I}\left\{a_{i j}+x_{i}-a_{i j} \cdot x_{i}\right\}=b_{j} ; \forall j \in J$ to satisfy the constraints. It is evident that at the $\mathrm{j}$-th equation we have $a_{i j}+x_{i}-a_{i j} \cdot x_{i} \leq \max \left\{a_{i j}+x_{i}-a_{i j} \cdot x_{i}\right\} ; \forall i \in I$. This leads to: $\quad a_{i j}+x_{i}-a_{i j} \cdot x_{i} \leq b_{j}$ or $\quad x_{i} \leq\left(b_{j}-a_{i j}\right) /\left(1-a_{i j}\right)$ due $\quad$ to $0 \leq x_{i} \leq 1, \quad$ if $a_{i j}>b_{j} ; \forall i \in I$ in the system (4), then $\left(b_{j}-a_{i j}\right) /\left(1-a_{i j}\right)<0$ thus $x_{i} \leq 0$ and no solution for $x \in X(A, b)$ can satisfy the $\mathrm{j}$-th equation in the system (4).

\subsection{Lemma 2}

If $a_{i j}=1$, then $x_{i}$ has no effect.

\subsubsection{Proof}

Noticing that $b_{j}-a_{i j} \geq 0$, we have $b_{j}=1$, as a result subjected to the system (4) $x_{i}=0$. By Lemmas 1 and 2, one can easily acquire if $b_{j}=0$ and $a_{i j}>0$ for $i \in I$ in Eq. (2), then the solution set $X(A, b)$ is empty. On the other hand, if $b_{j}=0$ and $a_{i j}=0$ for all $i \in I$, then the $\mathrm{j}$-th equation becomes:

$\max \left\{x_{i}+0-0\right\}=0$.

$i \in I$

Since $0 \leq x_{i} \leq 1$, xi should be zero for all $i \in I$, if any solution exists in $X(A, b)$. Therefore, this study assumes: $b_{j}>0, \forall j \in J ; X(A, b) \neq \varnothing ; a_{i j}<b_{j}$ and $a_{i j}<1$ for all $i \in I, \forall j \in J$.

According to and Higashi \& Klir [12], once $X(A, b) \neq \varnothing$, the optimum points can be completely determined by a maximum and a finite number of minimum solutions. The maximum solution of the fuzzy relation equations with max-as composition can be easily derived by applying the following operation (Khorram et al. [15]):

$\hat{X}=A \mathrm{ob}=\left[\min _{\mathrm{j} \in \mathrm{J}}\left(a_{i j} \mathrm{ob}_{\mathrm{j}}\right)\right]_{i \in I}$

Where $a_{i j} \mathrm{ob}_{\mathrm{j}}:=\left(\begin{array}{ccc}1 & \text { if } & b_{j}=1 \\ \frac{b_{j}-a_{i j}}{1-a_{i j}} & \text { if } & b_{j}<1\end{array}\right.$

\subsection{Definition 1}

For any solution $x=\left(x_{i}\right)_{i \in I} \in X(A, b)$ in the system (4), $x_{i}$ is the binding variable if $a_{i j}+x_{i}-a_{i j} \cdot x_{i}=b_{j}$ holds for some $j \in J$. 
The set $J\left(x_{i}\right):=\left\{j \mid a_{i j}+x_{i}-a_{i j} \cdot x_{i}=b_{j} ; \forall j \in J\right\}$ denotes the binding set for $x_{i}$.

\subsection{Lemma 3}

Let $\hat{x}=\left(x_{i}\right)_{i \in I}$ be the maximum solution and $x=\left(x_{i}\right)_{i \in I}$ the solution of in the system (4); if $x_{i}$ is binding in the $\mathrm{j}$-th equation, then $\hat{x}_{i}$ is also binding there.

\subsubsection{Proof}

For any solution of $x=\left(x_{i}\right)_{i \in I} \in X(A, b)$, there are $\operatorname{Max}_{i \in I}\left\{a_{i j}+x_{i}-a_{i j} \cdot x_{i}\right\}=b_{j}, \forall j \in J$. Hence, $a_{i j}+x_{i}-a_{i j} \cdot x_{i} \leq b_{j}$ holds for any value of variable $x_{i}$, implying that $a_{i j}+\widehat{x}_{i}-a_{i j} \cdot \widehat{x}_{i} \leq b_{j} ; \forall j \in J$. Now, if $x_{i}$ is binding in the $\mathrm{j}$-th equation, then $a_{i j}+x_{i}-a_{i j} \cdot x_{i} \leq b_{j} ; \forall j \in J$. Moreover, $x_{i} \leq \hat{x}_{i}$ exists for any solution of $X$. Therefore, the following inequality will be derived:

$b_{j}=a_{i j}+x_{i}-a_{i j} \cdot x_{i} \leq a_{i j}+\hat{x}_{i}-a_{i j} \cdot \hat{x}_{i} \leq b_{j}$ :

This result suggests $a_{i j}+\widehat{x}_{i}-a_{i j} \cdot \widehat{x}_{i}=b_{j}$. Hence, $\widehat{x}_{i}$ is also binding in the $\mathrm{j}$-th equation.

\subsection{Theorem 1}

For any optimal solution, $x \in X(A, b)$, if $x_{i}$ is a binding variable, then $x_{i}=\hat{x}_{i}$.

\subsubsection{Proof}

For any solution of $x=\left(x_{i}\right)_{i \in I} \in X(A, b)$, there are $\operatorname{Max}\left\{a_{i j}+x_{i}-a_{i j} \cdot x_{i}\right\}=b_{j}$; $\forall j \in J$ since $x_{i}$ is a binding variable, $a_{i j}+x_{i}-a_{i j} \cdot x_{i}=b_{j}$ for some $j \in J$. By Lemma (5), $\hat{x}_{i}$ is also binding at the $\mathrm{j}$-th equation. Hence, $a_{i j}+\widehat{x}_{i}-a_{i j} \cdot \hat{x}_{i}=b_{i}$. Now, consider $x_{i}<\hat{x}_{i}$, that implies $b_{j}=a_{i j}+x_{i}-a_{i j} \cdot x_{i}<a_{i j}+\hat{x}_{i}-a_{i j} \cdot \hat{x}_{i}=b_{j}$, which is impossible. Therefore $x_{i}=\bar{x}_{i}$.

\subsection{Theorem 2}

Let $\breve{x}$ be an optimal solution of problem (3)-(4). Therefore, $\breve{x}_{i}=0$ or $\breve{x}_{i}=\bar{x}_{i}$.

\subsubsection{Proof}

If $\breve{x}_{i}$ is not a binding variable, we can assign 0 to $\breve{x}_{i}$. On the other hand, if $\breve{x}_{i}$ is a binding variable, then $\breve{x}_{i}=\widehat{x}_{i}$ by Theorem 1 .

\subsection{Theorem 3}

Let $\hat{x}=\left(\widehat{x}_{i}\right)_{i \in I}$ as the maximum solution of Eq. (5). For any optimal solution of $\breve{x}=\left(\breve{x}_{i}\right)_{i \in I} \in X(A, b)$ of (3)-(4) problem, the $\mathrm{i}$-th component of $\breve{x}$ is one of the following situations: 
1) If $c_{i}<0$, then $\breve{x}_{i}=\widehat{x}_{i}$;

2) If $c_{i}>0$, then $\breve{x}_{i}=0$ or $\breve{x}_{i}=\widehat{x}_{i}$;

3) If $c_{i}=0$ and $x$ is a binding variable, then $\breve{x}_{i}=\widehat{x}_{i}$;

4) If $c_{i}=0$ and $x$ is not a binding variable, then $\breve{x}_{i}$ can be any value in $\left[0 ; \widehat{x}_{i}\right]$.

The proof of this theorem may be found in Wu [14].

\section{A NEW ALGORITHM FOR SOLVING (3)-(4) MODEL}

Based on the results obtained in section 2, an augmented matrix is employed in this study to solve the general case of (3)-(4) problem with a max-as composition. A new algorithm is suggested for finding an optimal solution using this augmented matrix. According to Theorem 2, each component of the optimal solution $\breve{x}$ can be composed of either $\breve{x}_{i}=0$ or $\breve{x}_{i}=\bar{x}_{i}$ for all $i \in I$. Furthermore, Theorem 1 shows that for any solution of $x=\left(x_{i}\right)_{i \in I} \in X(A, b)$, if $x_{i}$ be a binding variable, then $x_{i}=\hat{x}_{i}$. Based on these properties, the objective is to select proper binding variables in order to derive the optimal solution for (3)-(4) problem. The maximum solution $x=\left(x_{i}\right)_{i \in I}$ and binding set $J\left(\widehat{x}_{i}\right)$ provide useful information in searching for all binding variables. Hence, the search is limited to $J\left(\hat{x}_{i}\right)$ and the augmented matrix $A u g_{1}=\left(p_{i j}\right)$ for $i \in\{1,2, \ldots, m\}$ and $j \in\{1,2, \ldots, n+1\}$ is defined as:

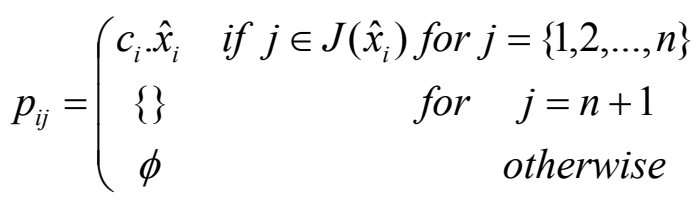

The numerical elements in the $\mathrm{i}$-th row of $\mathrm{Aug}_{1}$ correspond to the contributions in the objective function by setting $x_{i}=\bar{x}_{i}$. Notably, if $x_{i}=\hat{x}_{i}$ is not a binding variable but has the non-negative cost coefficient $c_{i} \geq 0$, then the elements in the i-th row of $\mathrm{Aug}_{1}$ are $p_{i j}=\phi ; \forall j \in J$. In the developed procedure for finding the optimal solution, the following index sets are denoted for matrix $\operatorname{Aug}_{1}$ :

$$
\begin{aligned}
& J_{i}\left\{A u g_{1}\right):=\left\{j \in\{1,2, \ldots, n\} \mid p_{i j}=c_{i} \cdot \widehat{x}_{i}\right\} \forall i \in I \text { and } \\
& I_{j}\left(A u g_{1}\right):=\left\{i \in I \mid p_{\mathrm{ij}}=c_{i} \cdot \widehat{x}_{i}\right\} \forall j \in\{1,2, \ldots, n\} .
\end{aligned}
$$

The index set $I_{j}\left(A u g_{1}\right)$ shows that the possible variables of $X$ may be selected as a binding variable or a nonbinding variable with a negative coefficient in the $j$-th equation. The augmented matrix $A u g_{2}=\left(q_{i j}\right)$ for $i \in\{1,2, \ldots, m\}$ and $j \in\{1,2, \ldots, n+1\}$ is defined as: 


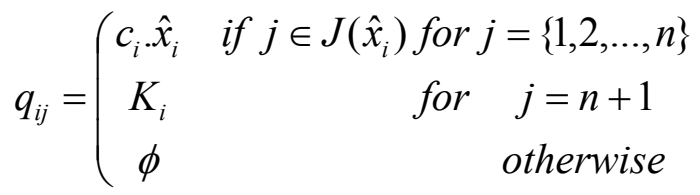

where for rows below the $\mathrm{i}$-th row of matrix $\mathrm{Aug}_{2}, K_{i}$ is defined as follows:

$K_{i}=\left\{s \in I \mid J_{s}\left(\right.\right.$ Aug $\left.\left._{2}\right) \subseteq J_{i}\left(A u g_{2}\right) ; i=1,2, . ., \vec{m}\right\}$;

$\vec{m}$ is the last row of the matrix. The following steps of the algorithm will be carried out to solve the problem:

1. Compute the vector $A \mathrm{o} \mathrm{b}$ by Eq. (5). Then control the consistency of the system (4) by verifying whether $\hat{x} \mathrm{O} A=\mathrm{b}$; if it is inconsistent, then stop. Otherwise, set the maximum solution $\widehat{x}=A \mathrm{o} b$.

2. Compute $I_{j}$ for each $j \in J$.

3. Generate the augmented value matrix $\mathrm{Aug}_{1}$.

4. From the (current) matrix $A u g_{1}$ compute the index sets $J_{i}=\left\{j \in J \mid p_{i j} \neq \phi\right\}$ for all variables $x_{i}$.

5. Arrange the rows of matrix values in descending order, (if necessary) as follows: $c_{i 1 j} \cdot \hat{x}_{i 1}>c_{i 2 j} \cdot \hat{x}_{i 2}>\ldots>c_{i m j} \cdot \hat{x}_{i m}$

6. If $p_{i j}<0$, then $x_{i}=\hat{x}_{i}$ and delete $\mathrm{i}$-th row and $\mathrm{j}$-th column. Repeat step 7 for all of the rows with $p_{i j}<0$.

7. (Generating the matrix $\mathrm{Aug}_{2}$ ) Update the augment column with equations (7).

Now, attribute the variables from the bottom upwards; therefore, each time go to the last row and repeat the following steps.

Run the following steps until all variables give an amount.

8. If $q_{i j}=\phi ; \forall j \in\{1,2, \ldots, n\}$ then $x_{i}=0$, and delete i-th row.

9. Let $x_{m}=\widehat{x}_{\vec{m}}$. Thus, delete $\widetilde{m}$-th rows and j-th columns for $\left\{\forall j \in J ; q_{\vec{m} j}>0\right\}$.

10. If $q_{\vec{m}(n+1)} \neq\{\}$, let $x_{s}=0$ for $s \in K_{\vec{m}}$.

11. If all variables reflect an amount, then stop. 


\section{NUMERICAL EXAMPLES}

In this section, two examples are considered.

\subsection{Example 1}

Consider the residents of three different regions. One tourism centre is located in each one of these areas. The tourism centre director of region $\mathrm{C}$ decides to cover the three regions as much as possible by improving the quality of tourism in his own region. He thinks that the following criteria may be useful to convince the residents of these three areas:

1. The prestige of region $\mathrm{C}$ as a tourism destination.

2. The traffic network, including air, rail, and road.

3. The tourism facilities and attractions.

He asks the centre's experts to determine the current status of tourism zone $C$, considering the above-mentioned criteria in each of the three regions. The experts prepare the membership matrix as follows:

$$
A=\left(\begin{array}{lll}
.2 & .4 & .3 \\
.4 & .3 & .4 \\
.4 & .55 & .4
\end{array}\right)
$$

where " $a_{i j}$ " indicates the membership of $\mathrm{i}$-th criteria in $\mathrm{j}$-th region. For instance, the prestige of region $C$ for residents of region $A$ is 0.2 ; for region $B$ it is 0.4 ; and for residents in his own area $(C)$ it is 0.3 . Therefore the tourism prestige of region $C$ is lower in region $A$ than in the other regions.

The institute's director believes that if he can raise at least one of the above criteria in the areas $A, B$, and $C$ respectively, to $0.6,0.7$ and 0.6 (b vector), then region $C$ can gain the upper hand.

He believes that by doing the following activities, he can promote the quality of the criteria that will be considered.

1. Marketing and advertising: anticipating the tourists' needs and their varied requests, in which their satisfaction is the most important principle to be considered. $\left(x_{1}\right)$

2. Transit infrastructure and competition with neighbouring regions, especially in the tourism areas $\left(x_{2}\right)$

3. Tourism centres include hotels, cultural centres (amphitheatres, exhibitions, etc), sports centres (tennis courts, cycling and walking tracks, golf courts, etc), nature parks and amusement parks. $\left(x_{3}\right)$

$X=\left(x_{1}, x_{2}, x_{3}\right)$ shows the amounts by which each factor needs to upgrade. We have $\mathrm{c}=(4,7,5)$ where, $c_{i}$ represent the cost associated with variable $x_{i}$ for $i=1,2,3$. He would like to know how to expand these activities in order to become the main tourism centre among its competitors, at the minimum cost.

Optimisation model for this problem is as follows:

Consider $\min z=4 x_{1}+7 x_{2}+5 x_{3}$

Subject to: $x \mathrm{o} A=b, \quad x_{i} \in[0,1]$, 
Where $A=\left(\begin{array}{ccc}.2 & .4 & .3 \\ .4 & .3 & .4 \\ .4 & .55 & .4\end{array}\right)$

And $\quad b=\left(\begin{array}{lll}.6 & .7 & .6\end{array}\right)$

Steps 1 and 2:

The $\widehat{x}=\left(\frac{3}{7}, \frac{1}{3}, \frac{1}{3}\right)$ can be easily found by equation (5). Here, $I_{1}=\{2,3\}, I_{2}=\{3\}$ and $I_{3}=\{1,2,3\}$.

Step 3:

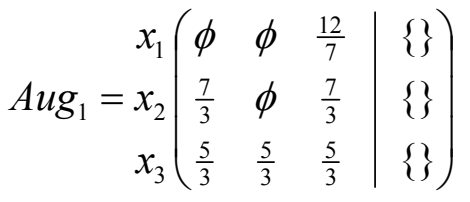

Step 4:

$J_{1}=\{3\}, J_{2}=\{1,3\}, J_{3}=\{1,2,3\}$.

Step 5 :

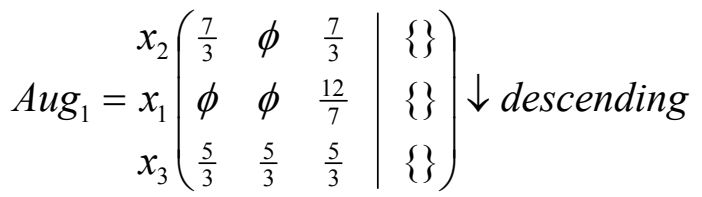

Step 6: not applicable in this example.

Step 7:

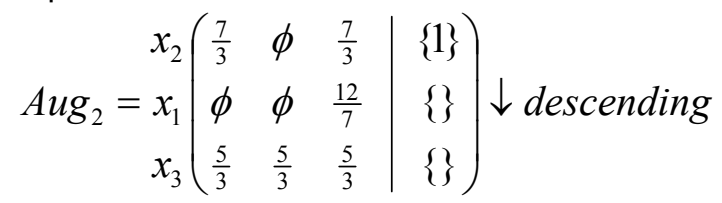

For $x_{2}: K_{2}=\{1\}$; because $J_{1} \subseteq J_{2}$.

Now, attribute the variables from bottom to top. Therefore, in each step, go to the last row and assign a value to the related variable.

Step 8: not applicable in this example.

Step 9: $x_{3}=\widehat{x}_{3}=\frac{1}{3}$ delete 3 -th rows and $\mathrm{j}$-th columns for $\left\{\forall j \in J ; q_{3 j}>0\right\}$.

Therefore, $x_{1}=0$ and $x_{2}=0$. Then $\breve{x}=\left(0,0, \frac{1}{3}\right)$. Thus the institute director must upgrade the third activity to 0.33 , to gain the upper hand among the regions. 


\subsection{Example 2}

Consider Min $z=2 \cdot x_{1}-4 \cdot x_{2}-x_{3}+5 \cdot x_{4}-3 \cdot x_{5}+x_{6}+7 x_{7}$

Subject to: $X \mathrm{oA}=\mathrm{b}$,

$$
x_{i} \in[0,1] \text {, }
$$

where $A=\left(\begin{array}{ccccc}\frac{1}{7} & \frac{3}{7} & \frac{2}{7} & \frac{1}{2} & \frac{1}{7} \\ 0 & \frac{1}{4} & \frac{1}{4} & \frac{1}{2} & 0 \\ \frac{1}{4} & \frac{1}{6} & \frac{2}{5} & \frac{1}{2} & \frac{1}{3} \\ \frac{1}{5} & \frac{2}{5} & \frac{1}{3} & \frac{4}{7} & \frac{1}{4} \\ \frac{1}{6} & \frac{1}{4} & \frac{1}{7} & \frac{5}{8} & \frac{1}{5} \\ \frac{1}{4} & \frac{1}{5} & \frac{3}{8} & \frac{5}{8} & \frac{1}{5} \\ \frac{1}{5} & \frac{7}{15} & \frac{1}{15} & \frac{3}{5} & \frac{1}{3}\end{array}\right)$

and $b=\left(\begin{array}{lllll}.4 & .6 & .5 & .7 & .4\end{array}\right)$

Step 1: First, the $\hat{x}=(.3, .33, .1, .2, .2, .2, .1)$ can easily be found.

Step 2:

$I_{1}=\{1,6\}, I_{2}=\{1\}, I_{3}=\{1,2,6\}, I_{4}=\{5,6\}, I_{5}=\{1,3,4,7\}$.

Step 3: The augmented value matrix $\left(A u g_{1}\right)$ :

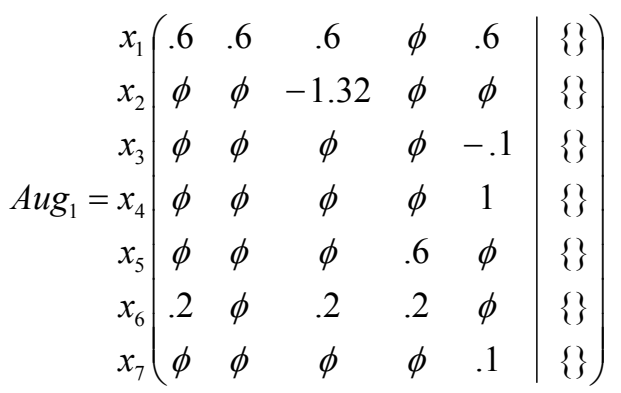

Step 4:

$J_{1}=\{1,2,3,5\}, J_{2}=\{3\}, J_{3}=\{5\}, J_{4}=\{5\}, J_{5}=\{4\}, J_{6}=\{1,3,4\}, J_{7}=\{5\}$. 
Step 5:

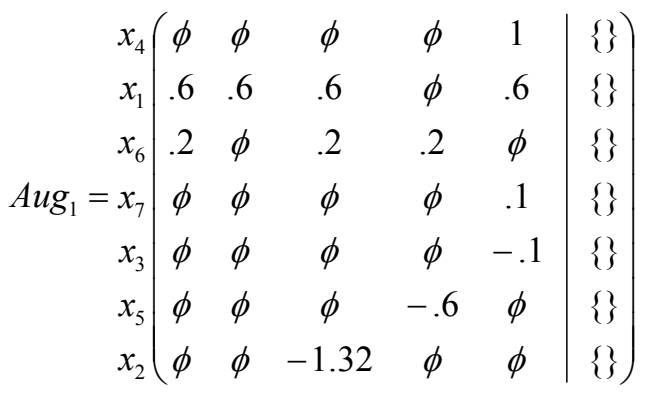

Step 6: For some $j \in J, p_{i j} \leq 0 ; i \in\{2,3,5\} ; x_{2}=\widehat{x}_{2}=.33 ; x_{3}=\widehat{x}_{3}=.1$;

$x_{5}=\widehat{x}_{5}=.2$.

Delete $\mathrm{i}$-th rows and $\mathrm{j}$-th columns for $\left\{\forall j \in J ; m_{\vec{m} j}<0\right\}$

Step 7: The matrix will be:

$$
\left.\operatorname{Aug}_{2}=\begin{array}{c|cc|c}
x_{4} & \phi & \phi & \{\} \\
x_{1} & .6 & .6 & \{6\} \\
x_{6} & .2 & \phi & \{\} \\
x_{7} & \phi & \phi & \{\}
\end{array}\right)
$$

Step 8: According to the step 8 of the algorithm, mentioned in section $3, x_{4}=0 ; x_{7}=0$.

Step 9: $x_{6}=\widehat{x}_{6}=.2$;

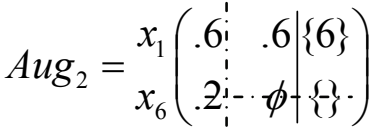

$$
\begin{aligned}
& x_{1}=\widehat{x}_{1}=.3
\end{aligned}
$$

Step 10: Where $K_{1}=\{6\}$ so: $x_{6}$ is 0 ;

Thus, $\breve{x}=(.3, .33, .1,0, .2,0,0)$ with an objective value $z(\breve{x})=-1.42$.

\subsection{Remark}

The numerical example has been solved by using the example data of Khorram et al. [15], and the solution has been obtained as follows:

$\breve{x}=(.3, .33,1,0, .2, .2,0)$

The corresponding objective value for solution $\breve{x}$ is -1.22 - obviously not the optimal solution. In this paper, to obtain a feasible solution, two elements of the matrix are changed as below:

$$
A_{32}: \frac{5}{6} \rightarrow \frac{1}{6} ; A_{33}: \frac{3}{5} \rightarrow \frac{2}{5} \text {. }
$$


The variable $x_{3}$ is nonbinding in both the second and the third equations. Therefore this change has no effect on the final solution.

\section{CONCLUSIONS}

In this paper, the maximum points are used for determining the minimum points after considering the feasible solution space. In the developed algorithm, it is not necessary to decompose the problem into two sub-problems, nor to use some rules to reduce the problem size.

\section{REFERENCES}

[1] Adamopoulos, G.I. \& Pappis, C.P. 1993. Some result on the resolution of fuzzy relation equation, Fuzzy Sets and Systems, 60, pp 83-88.

[2] Aliasing, K.P. 1986. Fuzzy set theory in medical diagnosis, IEEE Transactions on Systems, Man and Cybernetics, 16, pp 260-265.

[3] Bellman, R.E. \& Zadeh, L.A. 1970. Decision-making in fuzzy environment, Management Science, 17, pp 41-164.

[4] Di Nola, A. 1985. Relational equations in totally ordered lattices and their complete resolution, Journal de Mathematiques Pures et Appliquees, 107, pp 148-155.

[5] Zimmermann, H.J. 1999. Fuzzy set theory and its application, Kluwer Academic Publishers, Boston / Dordrecht / London.

[6] Pedrycz, W. 1981. An approach to the analysis of fuzzy systems, International Journal on Control, 34, pp 403-421.

[7] Dubois, D. \& Prade, H. 1980. Fuzzy sets and systems: Theory and applications, New York, Academic Press.

[8] Wenstop, F. 1976. Deductive verbal models of organizations, International Journal of Man-Machine Studies, 8, pp 93-311.

[9] Klir, G.J. \& Folger, T.A. 1988. Fuzzy sets, uncertainty, and information, PrenticeHall, Englewood Cliffs.

[10] Sanchez, E. 1976. Resolution of composite fuzzy relation equations, Information and Control, 30, pp 38-48.

[11] Di Nola, A., Sessa, S., Pedrycz, W. \& Sanchez, E. 1989. Fuzzy relation equations and their applications to knowledge engineering, Dordrecht, Kluwer Academic Press.

[12] Higashi, M. \& Klir, G.J. 1984. Resolution of finite fuzzy relation equations, Fuzzy Sets and Systems, 13, pp 65-82.

[13] Wu, Y.-K. \& Guu, S.-M. 2005. Minimizing a linear function under a fuzzy max-min relational equation constraint, Fuzzy Sets and Systems, 150, pp 147-162.

[14] Wu, Y.-K. 2007. Optimization of fuzzy relational equations With max-av composition, Journal of Information Sciences, 177, pp 4216-4229.

[15] Khorram, E., Ghodousian, A. \& Molai, A. 2006. Solving linear optimization problems with max-star composition equation constraints, Applied Mathematics and Computation, 179, pp 654- 661. 